\title{
Identifying Potential Hosts of Short-Branch Microsporidia
}

\author{
Annemie Doliwa ${ }^{1}$ (D) - Micah Dunthorn ${ }^{1,2}$ (D) $\cdot$ Erika Rassoshanska ${ }^{1}$ (D) Frédéric Mahé $^{3,4}$ (D) David Bass ${ }^{5,6,7}$ (D) \\ Camila Duarte Ritter ${ }^{1}$
}

Received: 14 August 2020 / Accepted: 3 December 2020 / Published online: 9 January 2021

(C) The Author(s) 2021, corrected publication 2021

\begin{abstract}
Microsporidia are obligate parasites that are closely related to Fungi. While the widely known "long-branch" Microsporidia infect mostly metazoans, the hosts of "short-branch" Microsporidia are only partially characterized or not known at all. Here, we used network analyses from Neotropical rainforest soil metabarcoding data, to infer co-occurrences between environmental lineages of short-branch microsporidians and their potential hosts. We found significant co-occurrences with several taxa, especially with Apicomplexa, Cercozoa, and Fungi, as well as some Metazoa. Our results are the first step to identify potential hosts of the environmental lineages of short-branch microsporidians, which can be targeted in future molecular and microscopic studies.
\end{abstract}

Keywords Neotropics $\cdot$ Network analyses $\cdot$ Parasites $\cdot$ Protists $\cdot$ Soil biodiversity

Environmental DNA sequencing studies have uncovered numerous protistan parasitic groups in different environments. For example, apicomplexans dominate soils in Neotropical rainforests [1] and Syndiniales are likewise species-rich in marine waters [2]. At least at larger taxonomic levels, it is relatively straightforward to infer the hosts of these protistan parasites: the apicomplexans mostly infect metazoans [3] and the Syndiniales infect metazoans and other protists [4]. However, we do not always know so clearly who are the hosts for other protistan parasite groups uncovered in environmental

Camila Duarte Ritter

camila.duarte-ritter@uni-due.de; kmicaduarte@gmail.com

1 Eukaryotic Microbiology, University of Duisburg-Essen, Universitätsstrasse 5, S05 R04 H83, 45141 Essen, Germany

2 Centre for Water and Environmental Research (ZWU), University of Duisburg-Essen, 45141 Essen, Germany

3 CIRAD, UMR BGPI, F-34398 Montpellier, France

4 BGPI, Université de Montpellier, CIRAD, IRD, Montpellier SupAgro, Montpellier, France

5 Centre for Environment, Aquaculture and Fisheries Science (Cefas), Barrack Road, Weymouth, Dorset DT4 8UB, UK

6 Department of Life Sciences, The Natural History Museum, Cromwell Road, London SW7 5BD, UK

7 Sustainable Aquaculture Futures, University of Exeter, Exeter EX4 4QD, UK
DNA sequencing studies. One such example of this lack of knowing who are the potential hosts are the "short-branch" microsporidians [5].

The short-branch microsporidians form a basal clade leading up to the more widely known "long-branch" Microsporidia [5]. Long-branch Microsporidia are mostly parasites of metazoans [6], but some can infect ciliates and other protists [7]. While the long-branch Microsporidia have highly reduced genomes and complex polar filaments that allow the penetration of cells, the short-branch microsporidians have less reduced genomes and they lack fully developed polar filaments [5]. The short-branch microsporidians include the partially characterized Paramicrosporidium that are parasites of Saccamoeba limax [8] and Vannella [9], Mitosporidium that are parasites of the crustacean Daphnia [10], as well as Morellospora, an amoeba parasite from the same clade as Mitosporidium [11], and Nucleophaga, a parasite of Thecamoeba [12]. The short-branch microsporidians also include numerous environmental lineages recently uncovered in a re-analysis of a metabarcoding study of Neotropical rainforest soils [5] and other environments [5, 13]. Presumably all of these environmental lineages phylogenetically assigned to the short-branch microsporidians are likewise parasitic; it is unknown, though, who are their potential microbial- or macro-organismic hosts, or where to even begin to look for them in environments as species-rich as tropical forests. 
Fig. 1 Co-occurrence network with OTUs as nodes and correlations as edges; the node size illustrates the abundance of the OTU. Mitosporidian OTUs are highlighted in turquois and paramicrosporidian OTUs in red

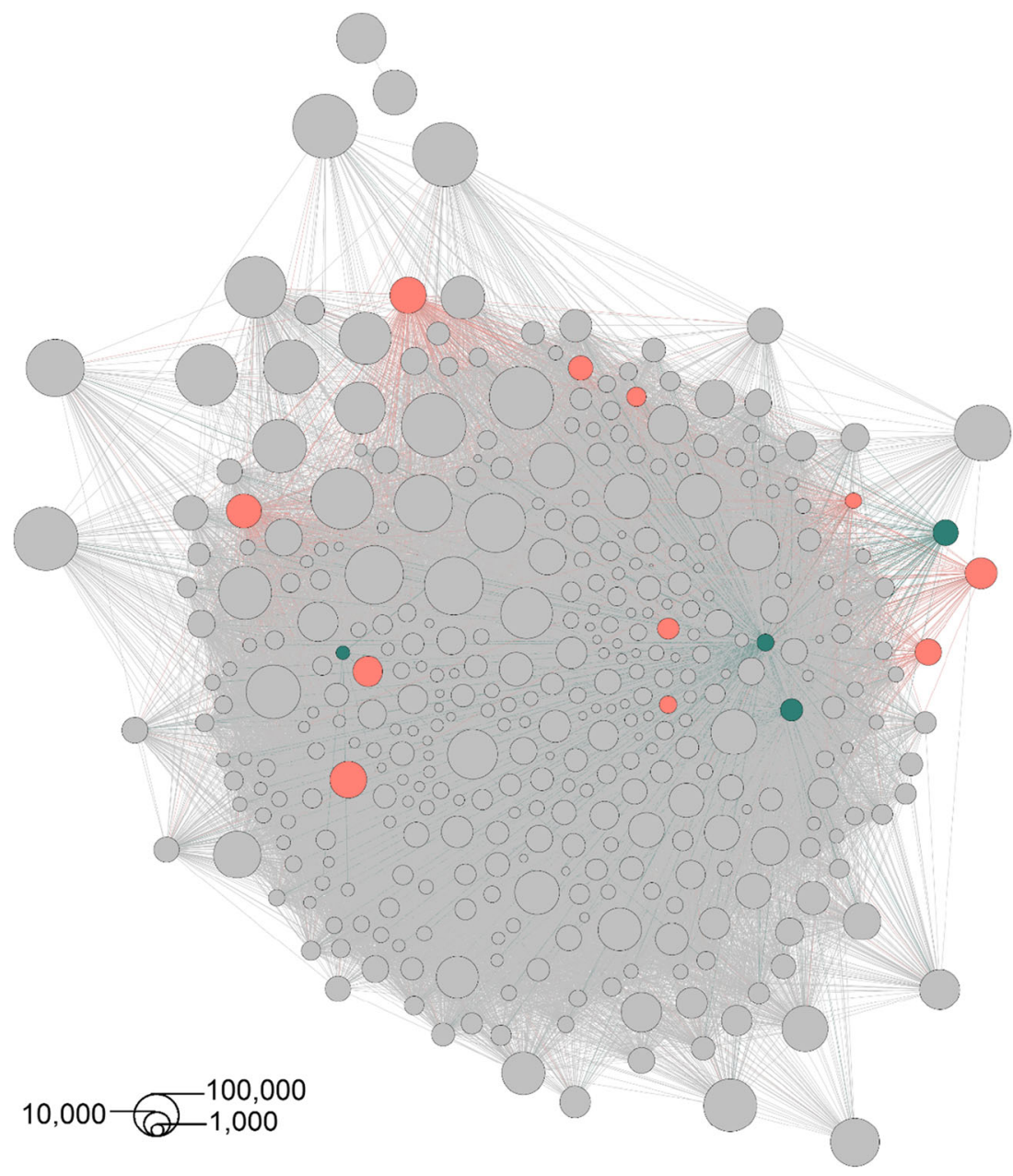

A novel approach to evaluating the diversity of protistan parasites and their hosts in metabarcoding datasets was recently demonstrated [14]. Using linear regression models, Singer et al. [14] showed that the abundances of apicomplexans and their metazoan hosts positively correlated across alpine sites in Switzerland. That type of analysis is dependent in part, though, on knowing what are the potential hosts through previous observations. Another approach to unravel potential host-parasite relationships when the hosts are unknown is to use co-occurrence network analyses. Although network analyses based on co-occurrences do not confirm biotic interactions [15], co-occurrence network can highlight potentially interesting taxonomic groups as potential hosts.

We used a co-occurrence network built from metabarcoding data from Mahé et al. [1]. Briefly, the data came from soils collected in lowland rainforests in Costa Rica, Panama, and Ecuador. The soils were amplified using broad eukaryotic primers for the V4 region of SSU-rRNA [16] and sequenced with Illumina MiSeq. After initial cleaning steps, reads were clustered into operational taxonomic units (OTUs) with swarm [17] and taxonomically assigned using the $\mathrm{PR}^{2}$ database [18]. Most of the OTUs were assigned to different protistan taxa, while others were assigned to Fungi and Metazoa. From this original data, refinements of the taxonomic assignments placed 974 OTUs into the short-branch microsporidia [5]. We calculated the richness of these OTUs with vegan v.2.5-6 [19], and compared the exclusive and shared OTUs by country with a Venn diagram [20] in R v.3.6.3 [21].

Representative sequences from all eukaryotic OTUs were used to construct a co-occurrence network with the NetworkNullHPC script (https://github.com/lentendu/ NetworkNullHPC). In this network, the OTUs are represented as nodes, and a statistically significant Spearman correlation between two OTUs is represented by an edge between them. The network contains only OTUs with a significant co-occurrence with at least one other OTU, using a set of null models following Connor et al. [22]. The resulting co-occurrence matrix was analyzed in $\mathrm{R}$ with tidyverse v.1.3.0 [23] and igraph v.1.2.4.2 [24], then explored and visualized with Gephi v.0.9.2 [25] using the Yifan $\mathrm{Hu}$ layout. The network was filtered for short-branch microsporidians and their correlating nodes, then further explored with a Sankey diagram made in networkD3 v.0.4 
Fig. 2 Sankey diagram showing the edges between microsporidian OTUs (left) and their target OTUs (right) in the co-occurrence network. Edges with paramicrosporidian OTUs are marked in red, those with mitosporidian OTUs are colored as turquoise

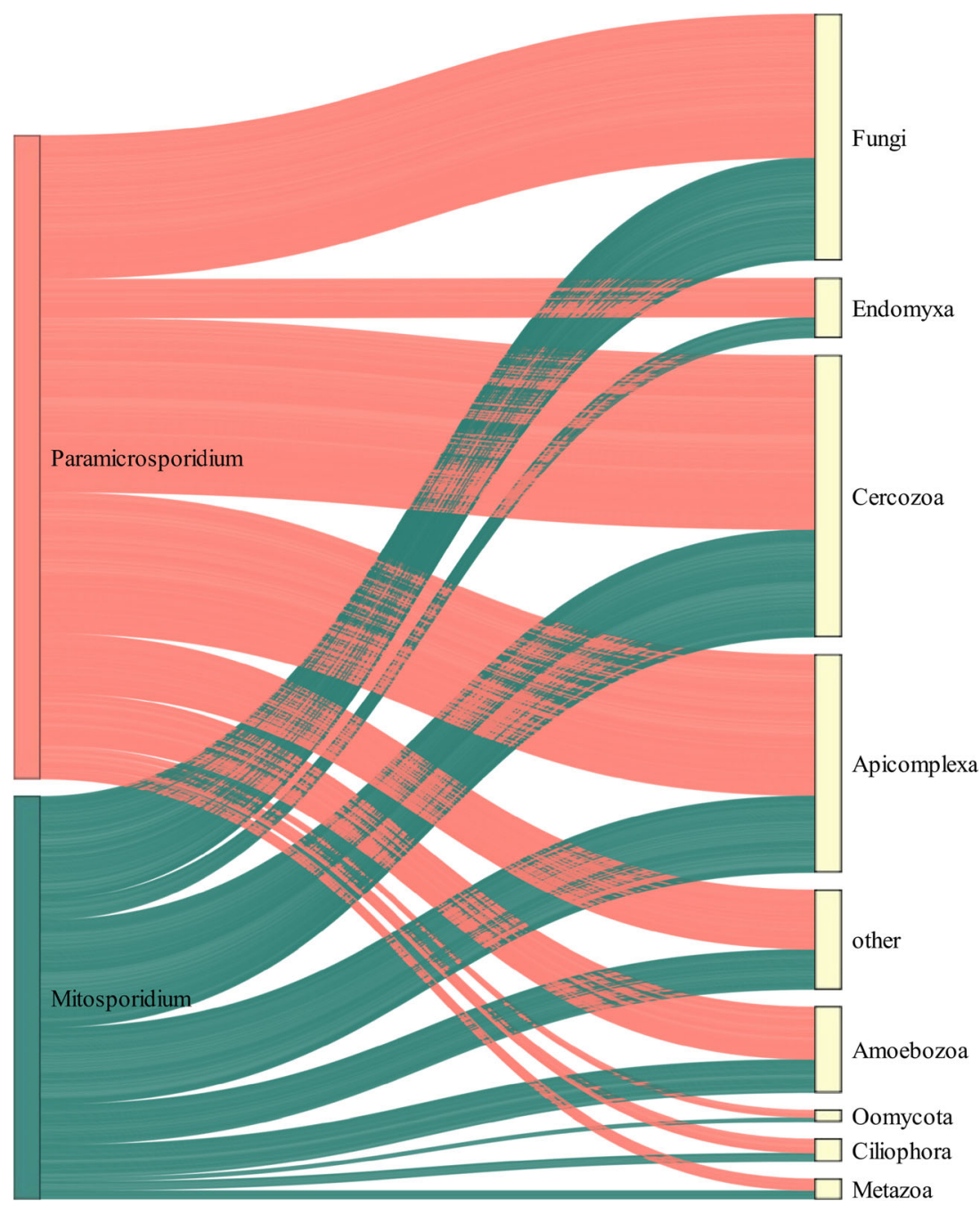

[26]. Spearman correlation was used as a value to link shortbranch microsporidia and their correlating nodes in the Sankey diagram as it is a ponderation between the number of edges and the strength of the correlation among nodes. Additionally, we ran networks analysis separatly for the 15 registered in our general network Microsporidia OTUs. For each of these Microposridia we just include plots that they are present (Fig. S1).

The co-occurrence network consisted of 14,329 edges involving 368 nodes (approximately $2.40 \%$ of all OTUs in the dataset). Costa Rica had the highest richness of short-branch Microsporidia, and the highest number of exclusive OTUs (Figs. S2 \& S3). However, just 15 widespread microsporidian OTUs were present in the network, corresponding to approximately $1.54 \%$ of all their OTUs in the dataset (Fig. 1). Of these OTUs, 11 had a closest taxonomic assignment to the Paramicrosporidium, and four to the Mitosporidium, although the OTUs likely form independent environmental lineages (Table S1). Filtering the co-occurrences for correlations only associated with these 15 short-branch microsporidian OTUs resulted in 1223 edges involving 244 nodes, with 768 edges belonging to OTUs assigned to the "paramicrosporidium" and 455 edges to OTUs assigned to the "mitosporidium" (Fig. 2; Tables S2 and S3). Although all known hosts from the studied clades are either Metazoa or Amoebozoa [8-10], the three most prominent groups co-occurring with the short-branch microsporidians are the Cercozoa, Fungi, and Apicomplexa. The two largest groups in the cercozoans to form cooccurrences were the largely bacterivorous testate amoebae in the Thecofilosea and Euglyphida. Within the Fungi, the largest groups were the Chytridiomycota and the Ascomycota, that are mostly found in those tropical soils in yeast-forming stages [27]. Most of the apicomplexans were in the Gregarinasina, which are parasites of invertebrates and dominated the soil protistan communities in the tropical forests [1]. Some other groups that also co-occurred with the shortbranch microsporidians included the already known hosts Amoebozoa and Metazoa, and also Endomyxa, Ciliophora, and Oomycota. The few metazoans in the networks were assigned to the Nematoda and Annelida (Table S4).

Although we found protists, fungi, and metazoans cooccurring with the short-branch microsporidians, the network 
analyses do not directly demonstrate that they are actual hosts. Co-occurrences can be inferred because of similar environment preferences, and actual biotic interactions may not have been inferred because the signal was too weak in the data [15]. Additionally, some of the co-occurrences here could have been inferred just because the cercozoans, fungi, and apicomplexans were extremely OTU-rich in the dataset. Potentially more of the short-branch microsporidians could have metazoan hosts, but the use of the SSU-rRNA environmental sequences likely underestimated their diversity, highlighted by the low proportion $(1.54 \%)$ of short-branch Microsporidia OTUs registered in our network. Yet, we inferred co-occurrences between four annelids and one nematode, which are known to be involved in host-parasite associations with long-branch Microsporidia [e.g., 28, 29].

Even in light of these potential limitations, the cooccurrence networks here highlight taxa that should be evaluated further for being the hosts of the environmental linages of short-branch microsporidians in complex Neotropical rainforest communities. These additional observations could include fluorescence in situ hybridization (FISH) probes designed for the short-branch microsporidians and used on cell isolates of cercozoans, fungi, apicomplexans, and possibly metazoan. Furthermore, the co-occurrence network approach proposed here can be used to identify potential hosts of other parasiticprotists uncovered in environmental DNA sequencing studies of in different complex environments.

Supplementary Information The online version contains supplementary material available at https://doi.org/10.1007/s00248-020-01657-9.

Acknowledgments We thank Dominik Foster for the help to run the cooccurrence network analysis.

Author Contributions C.D.R., A.D., and M.D. designed the study; C.D.R. and A.D. led all analyses with contributions from E.R.; F.M. and D.B. made the data available. A.D. led the writing of the manuscript with contributions from all authors.

Funding We received funding from the Alexander von Humboldt Foundation to C.D.R., and the Deutsche Forschungsgemeinschaft (\#DU1319/5-1) to M.D. Open Access funding enabled and organized by Projekt DEAL.

Data Availability The data is available at Mahé et al. (2017).

\section{Compliance with Ethical Standards}

Competing Interests The authors declare that they have no competing interests.

Ethics Approval No ethical approbation was necessary to conduct this research.
Open Access This article is licensed under a Creative Commons Attribution 4.0 International License, which permits use, sharing, adaptation, distribution and reproduction in any medium or format, as long as you give appropriate credit to the original author(s) and the source, provide a link to the Creative Commons licence, and indicate if changes were made. The images or other third party material in this article are included in the article's Creative Commons licence, unless indicated otherwise in a credit line to the material. If material is not included in the article's Creative Commons licence and your intended use is not permitted by statutory regulation or exceeds the permitted use, you will need to obtain permission directly from the copyright holder. To view a copy of this licence, visit http://creativecommons.org/licenses/by/4.0/.

\section{References}

1. Mahé F, De Vargas C, Bass D et al (2017) Parasites dominate hyperdiverse soil protist communities in Neotropical rainforests. Nat Ecol Evol 1:1-8. https://doi.org/10.1038/s41559-017-0091

2. De Vargas C, Audic S, Henry N et al (2015) Eukaryotic plankton diversity in the sunlit ocean. Science 348(6237):1261605. https:// doi.org/10.1126/science.1261498

3. Votýpka J, Modrý D, Oborník M, Šlapeta J, Lukeš J (2017) Apicomplexa. In: Archibald JM, Simpson AGB, Slamovits CH (eds) Handbook of the Protists, Second. Springer, New York, pp 567-624

4. Guillou L, Viprey M, Chambouvet A, Welsh RM, Kirkham AR, Massana R, Scanlan DJ, Worden AZ (2008) Widespread occurrence and genetic diversity of marine parasitoids belonging to Syndiniales (Alveolata). Environ Microbiol 10:3349-3365

5. Bass D, Czech L, Williams BAP, Berney C, Dunthorn M, Mahé F, Torruella G, Stentiford GD, Williams TA (2018) Clarifying the relationships between microsporidia and cryptomycota. J Eukaryot Microbiol 65:773-782. https://doi.org/10.1111/jeu.12519

6. Cali A, Becnel JJ, Takvorian PM (2017) Microsporidia. In: Archibald JM, Simpson AGB, Slamovits CH (eds) Handbook of the Protists, Second. Springer, New York, pp 1559-1618

7. Fokin SI, Di Giuseppe G, Erra F, Dini F (2008) Euplotespora binucleate n. gen., n. sp.(Protozoa: Microsporidia), a parasite infecting the hypotrichous ciliate Euplotes woodruffi, with observations on microsporidian infections in Ciliophora. J Eukaryot Microbiol 55:214-228

8. Michel R, Müller K-D, Hauröder B (2009) A novel microsporidian endoparasite replicating within the nucleus of Saccamoeba limax isolated from a pond. Endocytobios Cell Res 19:120-126

9. Michel R, Schmid EN, BoÈker T et al (2000) Vannella sp. harboring Microsporidia-like organisms isolated from the contact lens and inflamed eye of a female keratitis patient. Parasitol Res 86:514-520

10. Haag KL, James TY, Pombert J-F, Larsson R, Schaer TMM, Refardt D, Ebert D (2014) Evolution of a morphological novelty occurred before genome compaction in a lineage of extreme parasites. Proc Natl Acad Sci 111:15480-15485

11. Corsaro D, Walochnik J, Venditti D et al (2020) Solving an old enigma: Morellospora saccamoebae gen. nov., sp. nov.(Rozellomycota), a Sphaerita-like parasite of free-living amoebae. Parasitol Res 119:1-10

12. Corsaro D, Walochnik J, Venditti D, Müller KD, Hauröder B, Michel R (2014) Rediscovery of Nucleophaga amoebae, a novel member of the Rozellomycota. Parasitol Res 113:4491-4498

13. Richardson E, Bass D, Smirnova A, Paoli L, Dunfield P, Dacks JB (2020) Phylogenetic estimation of community composition and novel eukaryotic lineages in Base Mine Lake: an oil sands tailings reclamation site in northern Alberta. J Eukaryot Microbiol 67:86-99 
14. Singer D, Duckert C, Heděnec $P$ et al (2020) High-throughput sequencing of litter and moss eDNA reveals a positive correlation between the diversity of Apicomplexa and their invertebrate hosts across alpine habitats. Soil Biol Biochem 147:107837

15. Blanchet FG, Cazelles K, Gravel D (2020) Co-occurrence is not evidence of ecological interactions. Ecol Lett 23:1050-1063. https://doi.org/10.1111/ele.13525

16. Stoeck T, Bass D, Nebel M et al (2010) Multiple marker parallel tag environmental DNA sequencing reveals a highly complex eukaryotic community in marine anoxic water. Mol Ecol 19:21-31

17. Mahé F, Rognes T, Quince C, de Vargas C, Dunthorn M (2015) Swarm v2: highly-scalable and high-resolution amplicon clustering. PeerJ 3

18. Guillou L, Bachar D, Audic S, Bass D, Berney C, Bittner L, Boutte C, Burgaud G, de Vargas C, Decelle J, del Campo J, Dolan JR, Dunthorn M, Edvardsen B, Holzmann M, Kooistra WHCF, Lara E, le Bescot N, Logares R, Mahé F, Massana R, Montresor M, Morard R, Not F, Pawlowski J, Probert I, Sauvadet AL, Siano R, Stoeck T, Vaulot D, Zimmermann P, Christen R (2013) The Protist Ribosomal Reference database (PR 2 ): a catalog of unicellular eukaryote small sub-unit rRNA sequences with curated taxonomy. Nucleic Acids Res 41:597-604. https://doi.org/10.1093/nar/ gks 1160

19. Oksanen J, Blanchet FG, Kindt R, et al (2010) Vegan: community ecology package. R package version 1.17-4. http://cranr-project. org $>$. Accessed 15 June 2020

20. Oliveros JC (2007) VENNY. An interactive tool for comparing lists with Venn Diagrams. https://bioinfogp.cnb.csic.es/tools/venny/ index.html. Accessed 21 July 2020
21. R Core Team (2003) R Core Team (2018). R: A language and environment for statistical computing. R Foundation for Statistical Computing, Vienna, Austria. URL https://www.R-project.org/. Accessed 15 June 2020

22. Connor N, Barberán A, Clauset A (2017) Using null models to infer microbial cooccurrence networks. PLoS One 12:1-23. https://doi. org/10.1371/journal.pone.0176751

23. Wickham H (2017) Tidyverse: easily install and load "Tidyverse" packages (version R package version 1.1. 1)

24. Csardi G, Nepusz T (2006) The igraph software package for complex network research. InterJournal, Complex Syst 1695:1-9

25. Bastian M, Heymann S, Jacomy M (2009) Gephi: an open source software for exploring and manipulating networks. Third international AAAI conference on weblogs and social media

26. Allaire JJ, Gandrud C, Russell K, Yetman CJ (2017) networkD3: D3 javascript network graphs from $\mathrm{r}$. R package version 04

27. Dunthorn M, Kauserud H, Bass D, Mayor J, Mahé F (2017) Yeasts dominate soil fungal communities in three lowland Neotropical rainforests. Environ Microbiol Rep 9:668-675. https://doi.org/10. $1111 / 1758-2229.12575$

28. Troemel ER, Félix M-A, Whiteman NK, Barrière A, Ausubel FM (2008) Microsporidia are natural intracellular parasites of the nematode Caenorhabditis elegans. PLoS Biol 6:e309

29. Oumouna M, El-Matbouli M, Hoffmann RW, Bouix G (2000) Electron microscopic study of a new microsporean Microsporidium epithelialis sp. n. infecting Tubifex sp.(Oligochaeta). Folia Parasitol (Praha) 47:257-265 